\title{
OCCUPATIONAL INFECTIONS
}

\author{
David Snashall
}

\section{Occupational groups at risk of infections contracted at work}

- Veterinary surgeons-leptospirosis, $\mathrm{Q}$ fever

- Farm workers-ringworm, leptospirosis, orf, tetanus, perhaps bovine spongiform encephalopathy

- Poultry workers-ornithosis, histoplasmosis, Newcastle disease

- Health workers-hepatitis, HIV

- Construction workers-tetanus

- Butchers and abattoir workers-Streptococcus suis, Q fever

- Forestry workers-Lyme disease

- Engineering workers-skin infection

- Overseas workers returning home-tropical diseases, brucellosis, anthrax

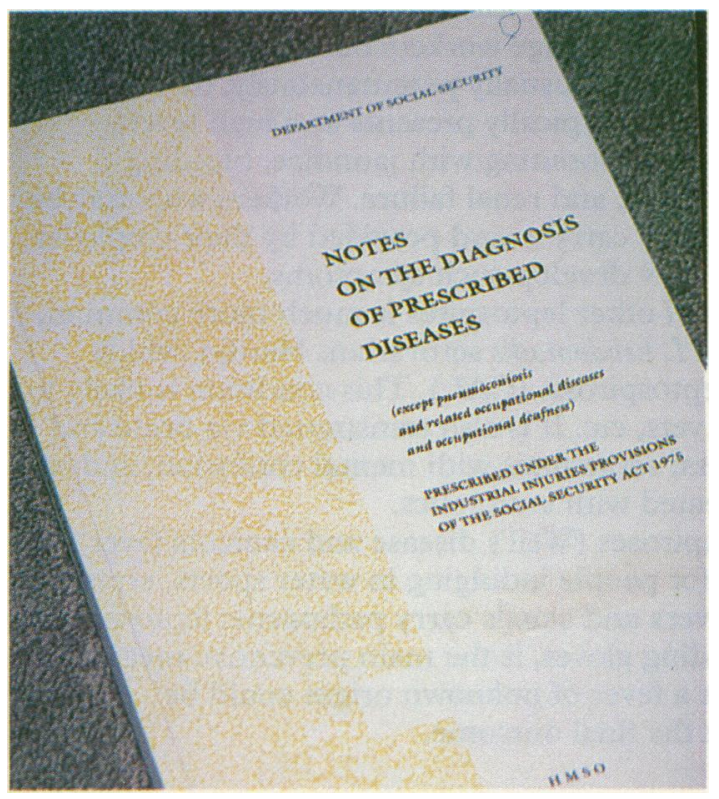

\section{Specific infections}

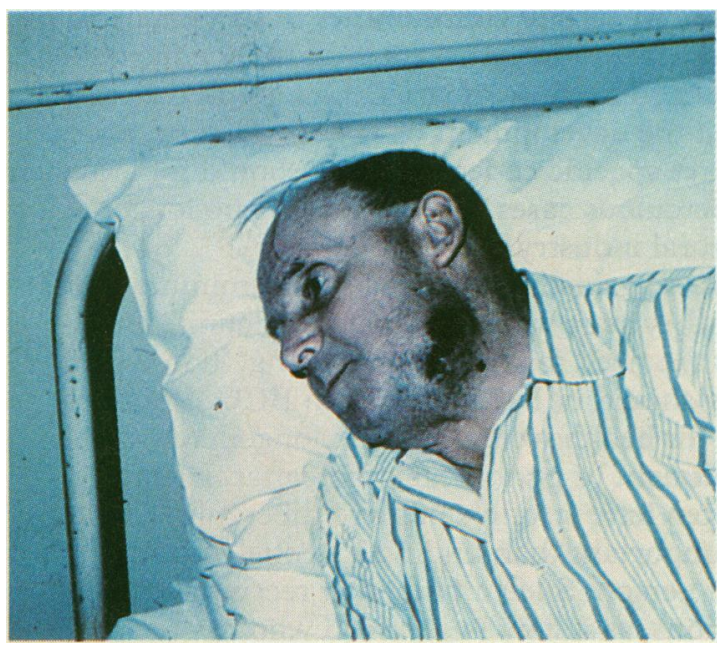

Patient with cutaneous anthrax.

\section{Anthrax}

Specific infections due to work are not common, but some systemic ones are serious and easy to miss unless there is a high index of suspicion. Carefully taking a patient's occupational history may reveal the diagnosis for an unusual illness. Superficial infections are less serious but may be difficult to diagnose and treat and can be transmitted to others. Some infections may cause an allergic response (such as farmer's lung), and endotoxins and mycotoxins can cause acute and chronic respiratory symptoms (such as mycobyssinosis in cotton workers). Like all occupational diseases, occupational infections are mostly preventable.

Some infections are included in the list of diseases prescribed under the Industrial Injuries Provisions of the Social Security Act 1975. The affected person can claim "no fault" compensation from the government if the "prescribed" disease is considered to be the result of certain kinds of work and if disability is rated at over $14 \%$.

There is a similar list of "reportable" diseases, some of them infectious, caused by work, and it is the duty of the employer (often advised by the doctor who diagnoses the disease) to report them under the RIDDOR regulations to the Health and Safety Executive or, sometimes, to the local environmental health department. They used to be called "notifiable" occupational diseases. The name changed in 1980 , and they should be distinguished from those infectious diseases that are notifiable under the Public Health Acts. Later this year CCDCs will start reporting occupational infections, publishing the numbers quarterly.

Employers are also bound to comply with the COSHH regulations when there is a risk of infectious disease associated with work. This demands a formal assessment of risk and the institution of precautions.

This is now rare in Britain and occurs in people who come into contact with animals infected with anthrax or who deal with animal products or residues (usually from abroad) that are contaminated with anthrax spores. The causative agent is Bacillus anthracis, and the disease usually presents as a black pustule or eschar on the skin at the site of primary infection. If not treated, this form of cutaneous anthrax leads to toxaemia and death. The organism is usually sensitive to penicillin. People who are most commonly affected are veterinary surgeons, butchers, slaughterers, and farm workers.

More severe forms of the disease-pulmonary or gastrointestinalare due to the inhalation or ingestion of anthrax spores. "Wool sorter's disease," the pulmonary form, is rapidly fatal and used to be seen in factories that dealt with imported wool or hides. Unsterilised bone meal used as a fertiliser is another source. Pulmonary anthrax remains a potential agent for germ warfare.

One or two cases of cutaneous anthrax have been notified annually since 1982

There have been no cases of wool sorter's disease in Britain since the 1930s. By contrast, Turkey, for example, reports 350 cases a year 


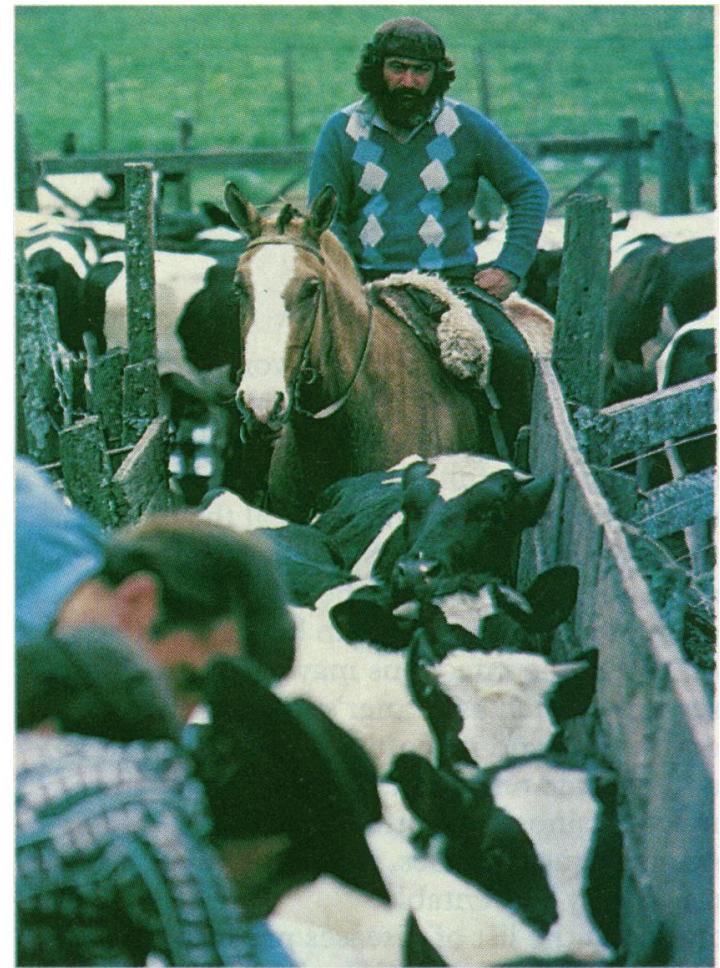

Farm workers are at increased risk of catching animal borne diseases.

Prevalence of leptospirosis (England
and Wales)
- No of cases of leptospirosis notified:
32 in 1989
20 in 1990
18 in 1993
- In $1989-90$ two patients received benefit for
occupationally acquired leptospirosis

Glanders and ankylostomiasis

These two infections no longer occur in Britain but may be imported from abroad. Glanders is systemic infection with Actinobacillus mallei, caught from horses. Ankylostomiasis is infection with hookworms of the genus Ancylostoma. These nematodes can survive and be transmitted during mining work and were once common in Cornish tin mines. The disease remains common in the tropics.

\section{Brucellosis}

Infection with Brucella abortus, which causes undulant fever, is also now a rarity in Britain due to the Ministry of Agriculture's crusade over the past 20 years to eradicate the disease in cattle by slaughtering and inoculation. Sufferers were usually farm workers and vets, with an occasional laboratory acquired infection. Infection with $B$ abortus (from cattle), $B$ melitensis (from sheep and goats), and $B$ suis (from pigs) is still common in many developing countries and should be suspected in people such as overseas aid workers in veterinary or farming jobs who return to Britain with fever and lymphadenopathy. It may also occur in foreign travelers who have consumed unpasteurised milk products.

\section{Leptospirosis}

Weil's disease, caused by Leptospira icterohaemorrhagiae, was once an important disease of canal and sewage workers but is now rare as an occupational disease. It is caught, usually percutaneously, by exposure to leptospires in rat's urine and typically presents as a high fever, headache, muscular pains, and vomiting with jaundice, bleeding disorders, hepatosplenomegaly, and renal failure. Workers who may be exposed to leptospires usually carry a card provided by their employer to show to their doctor if they develop such symptoms.

Infection with a variety of other leptospires is much more common, particularly infection with $L$ hebdomadis serovariant Hardjo, which causes cattle associated leptospirosis (CAL). This condition is likely to be seen in dairy workers, vets, etc. It is also transmitted via urine and presents as a flu-like illness, sometimes with mental confusion, and will run for months unless treated with antibiotics.

Water associated leptospiroses (Weil's disease and canicola fever) are also a recognised hazard for people indulging in water sports, especially canoeists. Most British rivers and canals carry pathogenic leptospires. Protective clothing, including gloves, is the main preventive measure, and early recognition that a fever of unknown origin could be leptospirosis will improve the final outcome.

\section{Tuberculosis}

As an occupationally acquired infection, tuberculosis mainly occurs in health workers. With the recent increase in cases of pulmonary tuberculosis worldwide and in certain communities in Britain, extra vigilance may be required. Laboratory workers used to face the highest risk, but this has now declined because of strict rules about processing tuberculous materials and the development of safe working practices. Necropsy workers also have specific codes that they should follow when dealing with possibly tuberculous cases. Tuberculosis is no longer a hazard in British agricultural industry.

To protect health workers against tuberculosis, their immune status should be checked when they start work or studies. A definite BCG vaccination scar obviates the need for tuberculin testing. Those with poor tuberculin reactivity should be vaccinated with BCG. Routine chest $x$ ray examinations for health workers are no longer considered necessary, either at recruitment or periodically thereafter. Health workers born overseas often have strongly positive tuberculin reactions, but this is generally less important than finding a strongly positive reaction in a British born person. Such a finding at recruitment may require further investigations, as it may in a person who has recently come from a country where tuberculosis is common.

Education of staff about the symptoms of tuberculosis is important, especially if they are likely to be dealing with patients who may have as yet undiagnosed pulmonary tuberculosis. Symptoms - coughing, sweats, and weight loss - must be investigated in the usual way. 
Advisory panels on hepatitis B and HIV infection have been set up by the Department of Health to help in the management of difficult cases among health workers

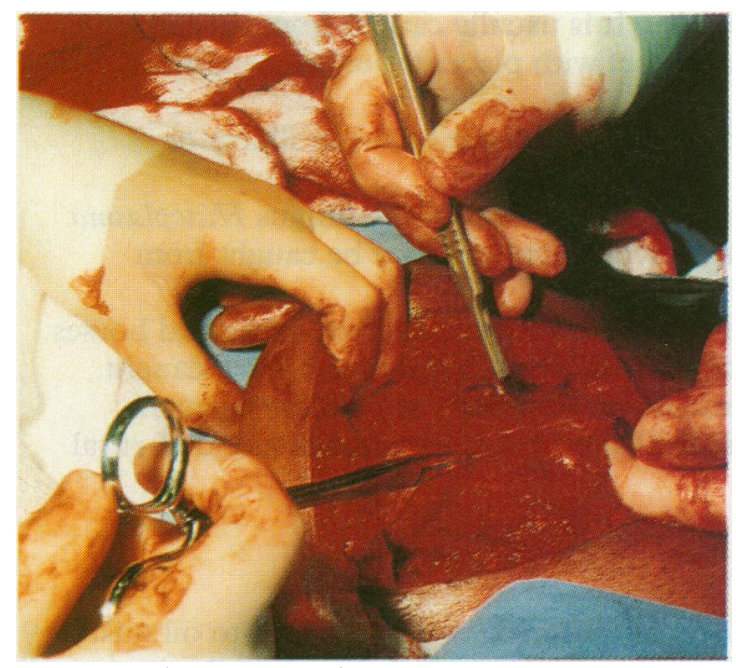

Health workers who perform exposure prone procedures should be vaccinated against hepatitis $B$.

Viral hepatitis

Hepatitis B and C are mainly a risk to health workers, although others such as prison warders and police officers may be exposed to hepatitis, usually blood transmitted. Hepatitis A, mainly spread by the faecal-oral route, has been transmitted by food handling procedures. There is a theoretical risk to sewage workers and hospital laboratory staff, but no excess numbers of cases have been recorded in these occupations.

Infection of health care workers may be with hepatitis A, B, or C or with various other agents-Epstein-Barr virus, cytomegalovirus, etc. One of the most important of these is hepatitis B, which represents a substantial risk to health workers, especially if they perform invasive or "exposure prone" procedures or if they work abroad in countries where the prevalence of the disease is high. Immunisation against hepatitis B is available, remarkably effective, and becoming cheaper. All medical students should be vaccinated before they start clinical work, as should other health workers, including dentists, who may be exposed to blood and body fluids, especially in uncontrolled circumstances such as accident and emergency work. It is doubtful whether the risk to other occupational groups warrants vaccination.

Recent guidelines from the Department of Health have emphasised the importance of vaccination against hepatitis B to protect health workers and their patients. Workers who perform exposure prone procedures (essentially deep surgery, blind needling, and dentistry) should, if they are not naturally immune or successfully vaccinated, be tested to exclude carriage of hepatitis B virus. If positive, they should be offered treatment or redeployed to safer work if they remain infectious. The incidence of hepatitis $B$ in health workers will decline as these procedures are taken up.

Chronic carriers of hepatitis B e antigen are infectious via their blood and body fluids and need only avoid performing exposure prone operations. They require expert confidential counselling about sexual contacts and possible redeployment and retraining. After a needlestick injury, a risk assessment must be made and a blood sample for serology may be required-for hepatitis B and, when relevant, for HIV and hepatitis $\mathrm{C}$.

Both hepatitis B and C have been transmitted from patients to health workers. No vaccine against hepatitis $C$ has yet been developed; health workers who have transmitted the disease should be barred from performing exposure prone procedures. Treatment with interferon alfa should be considered for chronic carriers of hepatitis B or C antigens.

\section{In the United Kingdom there were at least four definite cases of HIV transmission from patients to health workers during 1984-95}

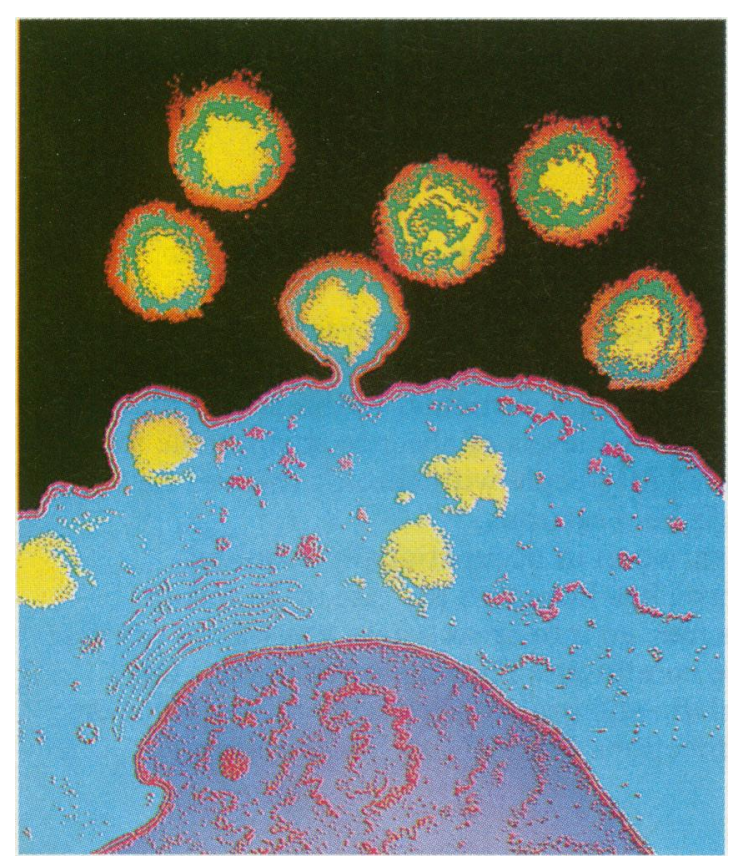

Enhanced, false colour electron micrograph showing HIV budding out of infected human T lymphocyte.

\section{HIV infection}

The HIV virus is transmitted in the same way as hepatitis B but with much greater difficulty. So far as is known (a transmission rate of $0.3 \%$ has been suggested) health workers have rarely been affected in this country. There are, however, around 60 reports worldwide of health workers who have apparently acquired HIV infection from professional contact with patients. These patients have usually been suffering from frank AIDS and a quantity of blood or other body fluid has been inoculated into the health worker, with subsequent seroconversion. Health care workers abroad may be at greater risk.

Protective measures are the same as for hepatitis B-use of universal precautions in handling blood and body fluids, care with needles, no resheathing of hollow needles, proper disposal of sharps, prompt first aid after sharps injuries, and proper reporting of such injuries.

The use of zidovudine as post-exposure prophylaxis is still controversial. The use of newer antiviral drugs in combination has also been recommended. To be effective, these drugs must be administered rapidly-within an hour of exposure if possible. A local policy on their use should be developed. Health workers who have suffered an injury that might have transmitted HIV need expert counselling and follow up testing for HIV antibody, preferably by an occupational health department or a doctor with experience of HIV infection. 


\section{Disease spread from farm animals}

Streptococcus suis causes an infection characterised by septicaemia, meningitis, possibly arthritis, pharyngitis, and diarrhoea. It is transmitted from seemingly healthy pigs and their carcases and thus may be a problem for butchers and slaughterers as well as pig breeders.

Avian chlamydiosis (ornithosis, psittacosis) usually presents as an atypical pneumonia and occurs when workers with turkeys, ducks, chickens, pigeons, and exotic birds inhale the dust of dried excreta or feathers. Vets, quarantine station workers, pet shop owners, and poultry workers may be affected.

Ovine chlamydiosis presents as an acute flu-like illness in vets, agricultural workers, shepherds, and abattoir workers who handle infected sheep. Abortion may occur in pregnant women.

$Q$ fever due to Coxiella burnetii causes a flu-like illness with possible pneumonia, pericarditis, myocarditis, and encephalitis. The chronic form may affect the aortic valve. It is usually caught from sheep, cattle, or goats, particularly from contact with placentas or unpasteurised milk.

Newcastle disease from fowls can also cause a flu-like illness or conjunctivitis.

Histoplasmosis, due to inhalation of spores of the fungus Histoplasma capsulatum, causes respiratory symptoms and may be caught from chicken manure.

Skin infections-Ringworm can be transmitted from cattle and horses. Orf (contagious pustular dermatitis) is a self limiting disease caught from sheep and usually presents on the hands as painless blisters. Hydatid disease is now rare in Britain but is sometimes found in rural communities.

\section{Non-agricultural diseases}

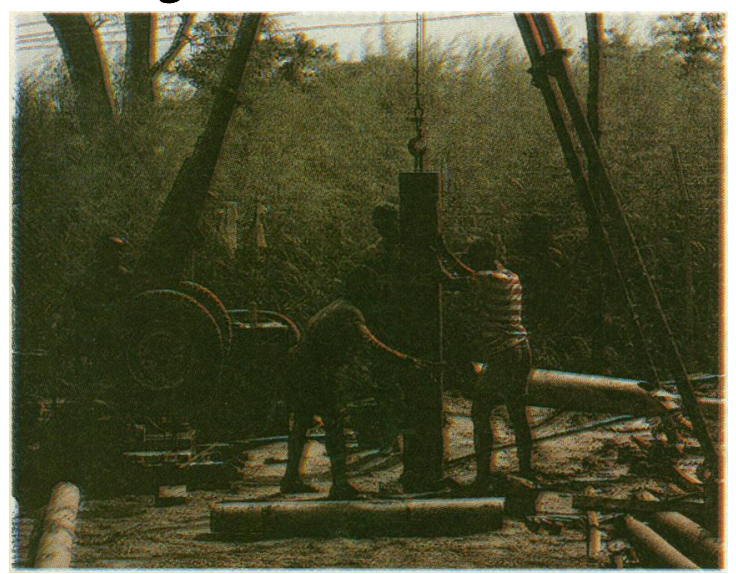

Workers in forested areas are at risk of acquiring viral haemorrhagic fevers.

\section{Immunisations}

\section{Occupations that require specific immunisations}

- Health workers - vaccination against rubella, hepatitis $B$, tuberculosis (BCG vaccine), and possibly chicken pox

- Construction workers, etc-vaccination against tetanus

- Agricultural workers-vaccination against tetanus.

- Veterinary surgeons-vaccination against tetanus and possibly rabies
The re-use of cutting oils in engineering works can lead to oil mists being contaminated with bacteria and fungi, and these have been implicated in various types of skin infection. Deep sea divers who use saturation techniques-in which they remain under increased barometric pressure for some weeks - are prone to otitis externa, especially with Pseudomonas species. Legionnellosis is sometimes occupationally acquired (see chapter on building related illnesses).

Viral haemorrhagic fevers, even yellow fever, can be occupational because they tend to be transmitted to agricultural or forest workers when they are clearing crops or trees. The diseases are sometimes imported and transmitted to health workers-Lassa fever, for example.

Forest workers may be at increased risk of Lyme disease. A bite from an infected tick transmits the causative organism Borrellia burgdorferi. Early symptoms are a red area around the bite, fever, joint pains, and headache. A variety of symptoms may supervene if the disease is not rapidly recognised and treated with antibiotics.

Because of their increased risk of infection, workers in certain occupations need specific immunisations.

Influenza vaccination is heavily advertised every year but is not indicated for healthy young workers, and the immunisation itself can cause some morbidity. Its efficacy in a particular outbreak is difficult to predict, and it is unlikely to reduce levels of absence attributable to sickness. It should be retained for people with chronic disease, for whom an attack of flu would be disastrous.

There is much regional variation in occupationally acquired infections. International travel has increased the rate of imported disease to Britain. Workers may go into areas where tourists do not visit and often experience worse conditions. They are often abroad for longer periods of time. Malaria still probably presents the commonest and most serious of such infections (see $A B C$ of Healthy Travel). 